\title{
Moya-moya disease: clinical and pathological report of a case with associated myopathy
}

\author{
H. B. C O A K H A M, L. W D U C H E N, A N D F. S CARA V I L L I \\ From the Departments of Neurosurgery and Neuropathology, The Maudsley Hospital and \\ Institute of Psychiatry, London
}

\begin{abstract}
SUMMARY A British born white man, aged 51 years, presented with cerebrovascular insufficiency, and was found radiologically to have moya-moya disease. He also suffered from a late onset type of progressive myopathy. Five months after the first signs of cerebrovascular disease he died of massive cerebellar infarction. At necropsy the brain showed multiple old and recent infarctions. The anterior cerebral, middle cerebral, and posterior communicating arteries showed segments of narrowing and occlusion by delicate connective tissue, with marked thickening and folding of the elastic lamina. Many dilated thin-walled vessels without a muscle layer and with a poorly formed elastic lamina were present in the subarachnoid space, these probably being newly formed collateral vessels. The cause of the occlusive lesions affecting the cerebral arteries is not known but the morphological appearances do not fall within any known aetiology. It seems that moya-moya disease could be regarded as a distinct entity.
\end{abstract}

The cerebrovascular syndrome known as moyamoya disease was first described from Japan in 1961 by Takeuchi. This condition usually affects the internal carotid arteries, causing occlusion distal to the origin of the ophthalmic artery, and it often involves the circle of Willis and main trunks of the cerebral arteries. An associated collateral system of fine vessels is present in the region of the basal ganglia, and collateral vessels also occur transdurally from external carotid branches.

It is now clear that moya-moya disease is not confined to the Japanese as was originally suggested (Kudo, 1968) since a number of cases have been reported from other parts of the world. The condition is also recognised as occurring infrequently in the UK although no published details of incidence are available. Indeed the disease is sufficiently uncommon outside Japan among Caucasians and Negroes that reports describe small numbers of cases or even single patients (Simon et al., 1968; Busch, 1969; Lepoire et al., 1969; Galligioni et al., 1971; O’Sullivan, 1973; Hoare and Keogh, 1974; Poór and Gács, 1974; Sogaard and Jorgensen, 1975; Meriwether et al.,

Address for reprint requests: Professor L. W. Duchen, Department of Neuropathology, Institute of Neurology, The National Hospital, Queen Square, London WCIN 3BG.

Accepted 2 October 1978
1976). This contrasts with the Japanese experience as illustrated by a series of 376 cases (see Kudo, 1971).

The Japanese cases fall into childhood and adult types, with peak incidences at 4-6 years and the fourth decade. Children present mainly with hemiparesis while subarachnoid haemorrhage is a common presenting feature in adults, because of leakage from leptomeningeal collateral vessels.

The name moya-moya, meaning "haze" or "puff of smoke," is used to describe the angiographic appearance of the extensive collateral network which is characteristic of this condition. However, the primary lesion is that of vascular occlusion, and in most cases the nature of this process remains obscure. Most reports are confined to clinical and radiological observations, and only few pathological reports appear in the world literature. We wish to report a case of moya-moya disease in an adult who also suffered from late onset muscular dystrophy, a combination hitherto undescribed. The patient died as a result of cerebrovascular insufficiency and a detailed postmortem examination was made.

\section{Case report}

A 51 year old right handed Englishman presented with sudden onset of left sided visual field defect 
and symptoms of spatial disorientation, being unable to dress himself and losing his way in familiar surroundings. Over the previous four years he had developed progressive weakness of the proximal lower limb muscles, with symptoms including difficulty in rising from low chairs. There was no family history of cerebrovascular disease or disorders of muscle. A diagnosis of polymyositis had been made and treatment with prednisolone 10 to $15 \mathrm{mg}$ daily and cyclophosphamide $100 \mathrm{mg}$ daily given for about one year.

Physical examination on admission showed a thin normotensive man with normal cardiac rhythm and without evidence of peripheral vascular disease. There were no cardiac murmurs. $\mathrm{He}$ was alert with normal speech but with slight impairment of memory for recent events. Both clinical tests and formal psychological testing demonstrated a marked visuospatial deficit. Examination of the visual fields (Bjerrum screen) revealed a complete left homonymous hemianopia. The reflexes in the upper limbs were abnormally brisk but there was depression of the knee jerks. The plantar responses were flexor. There was no evidence of sensory impairment.

There was obvious wasting and weakness of the gluteal muscles, quadriceps, and hamstrings, and weakness of hip adduction and abduction. Less marked weakness was also found in the pectoral muscles, trapezius, and serratus anterior muscles. There was no clinical muscle fibrillation, muscle tenderness, or myotonia.

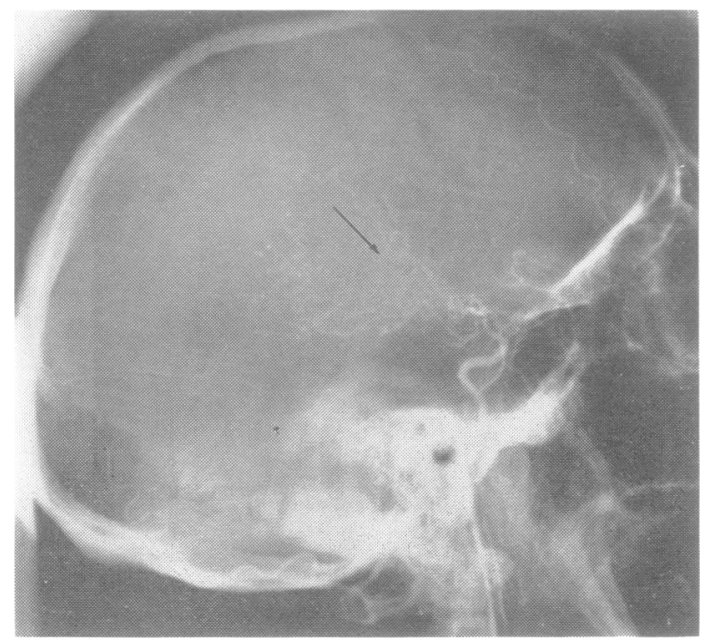

Fig. 1 Right carotid arteriogram shows narrowing of the distal part of the internal carotid artery with a leash of fine collateral vessels in the region of the basal ganglia (arrow).
INVESTIGATIONS

Isotope brain scanning ( ${ }^{3} \mathrm{Tc}$ ) demonstrated radionuclide accumulation in the right parieto-occipital region, which was also shown to be poorly perfused by a dynamic cerebral scan with the same isotope. The clinical impression was one of right parieto-occipital infarction.

\section{Angiography}

Right carotid angiography showed narrowing of the distal internal carotid artery and occlusion of the origins of anterior and middle cerebral arteries with a leash of fine collateral vessels in the region of the basal ganglia (Fig. 1). In a later phase distal segments of anterior and middle cerebral arteries were seen to fill via the collateral system (Fig. 2). The occipital and middle meningeal arteries were large and appeared to contribute to the collateral circulation. In the vertebral arteriogram fine anastomotic vessels were seen to pass forward to the parietal region and the thalamus (Fig. 3).

\section{Clinical tests}

A series of investigations of the possible causes of obliterative vascular disease were noncontributory. Investigations which yielded results within normal limits included haemoglobin $(14 \mathrm{~g} / \mathrm{dl})$; white cell and differential count; ESR $(5-12 \mathrm{~mm} / \mathrm{hr})$; blood glucose; plasma proteins including immunoglobulins; fasting plasma lipid profile; serum electrolytes and calcium; thyroid function; rheumatoid factor; LE cells; antinuclear factor; mitochondrial.

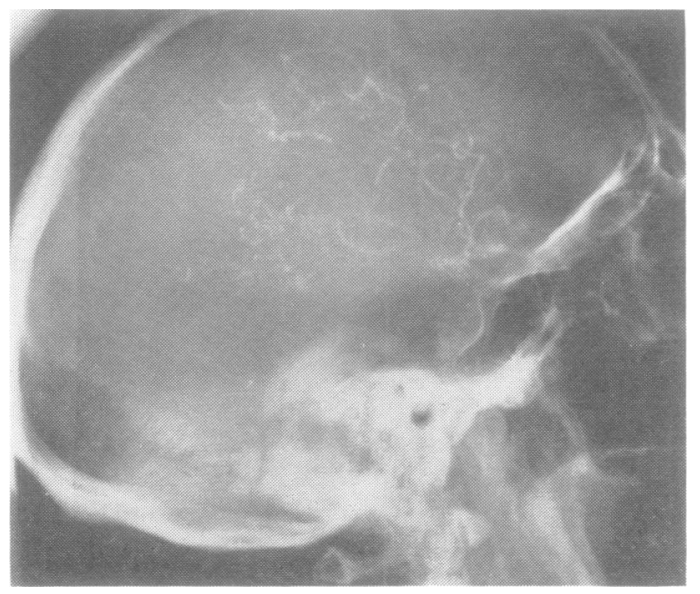

Fig. 2 A later phase of the right carotid arteriogram shows irregular filling of distal segments of anterior and middle cerebral arteries from the basal ganglion collaterals seen in Fig. 1. 


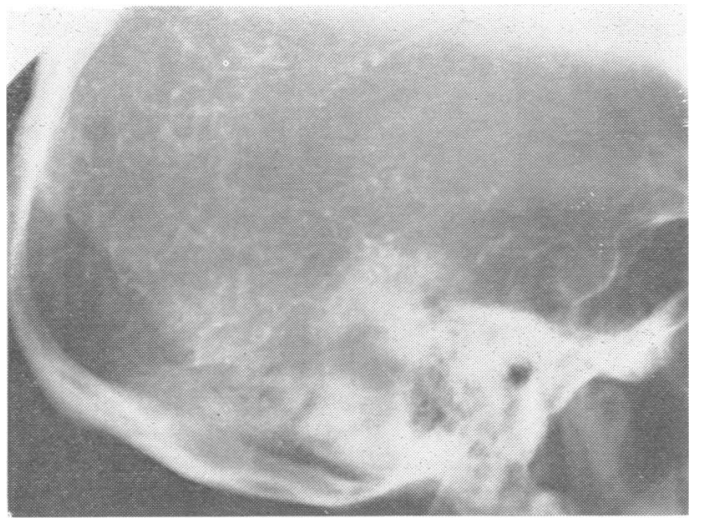

Fig. 3 Vertebral arteriogram showing fine anastomotic vessels passing forwards into middle cerebral artery territory and deeply into the basal ganglia.

thyroid and parietal cell antibodies. The WR was negative. Smooth muscle antibodies were present in low titre and were not considered significant. Serum creatine phosphokinase levels were persistently raised, between three and seven times the normal upper limit of $50 \mathrm{IU} / 1$, and the serum aldolase remained raised at about double the normal value of 0.5-3.1 IU/1. Enzyme levels did not respond to steroid or cyclophosphamide treatment, and the ESR before treatment was normal and remained so. There was no electrocardiographic abnormality.

\section{Electromyography}

Sampling of left quadriceps, tibialis anterior, deltoid, and abductor pollicis brevis showed abnormality of a mixed type (small amplitude and short duration polyphasic unit potentials, fibrillation, and "pseudo-myotonic" bursts of potentials) most marked in quadriceps and minimal in abductor pollicis brevis. Motor nerve conduction velocity (left lateral popliteal) was normal, and a normal sensory nerve action potential was recorded over the left sural nerve.

\section{Muscle biopsy}

The biopsy specimen was taken from the left quadriceps femoris and was studied in paraffin sections and with electron microscopy. Numerous necrotic muscle fibres were present, some showing a cellular reaction with invasion by myophages. There was great variation in fibre diameter and many fibres contained central nuclei. The picture was that of a primary myopathic condition-that is, dystrophy.
After investigation the hemianopia and nondominant parietal syndrome remained unchanged but the proximal limb girdle muscles became progressively weaker. The patient was discharged and was not seen again until his final admission five months later. At this time he developed severe occipital headache, became drowsy, and died after three days.

\section{Necropsy examination}

The body was that of a thin man with generalised atrophy of musculature. The skull and dura mater appeared normal. The leptomeninges showed a great increase in the number of capillaries over the whole brain, more marked on the right side (Fig. 4). There was extensive old softening and shrinkage of the gyri of the right occipital lobe, including the occipital pole. Both cerebellar hemispheres showed recent softening. The carotid and vertebral arteries were followed from their origin to their entry into the cranial cavity and were free of abnormalities. The circle of Willis was dissected out. Intermittent lengths of some of the components of the circle or of the main arteries at the base of the brain showed reduction of their calibre and were whitish in colour (Fig. 5). The vessels involved were the right anterior cerebral, both middle cerebral, and the left posterior communicating artery. Between these narrowed white regions the main vessels were free of atheroma. There was recent thrombus in the left vertebral artery.

Coronal slices showed moderate symmetrical dilatation of the ventricular system including the third ventricle. There was generalised prominence

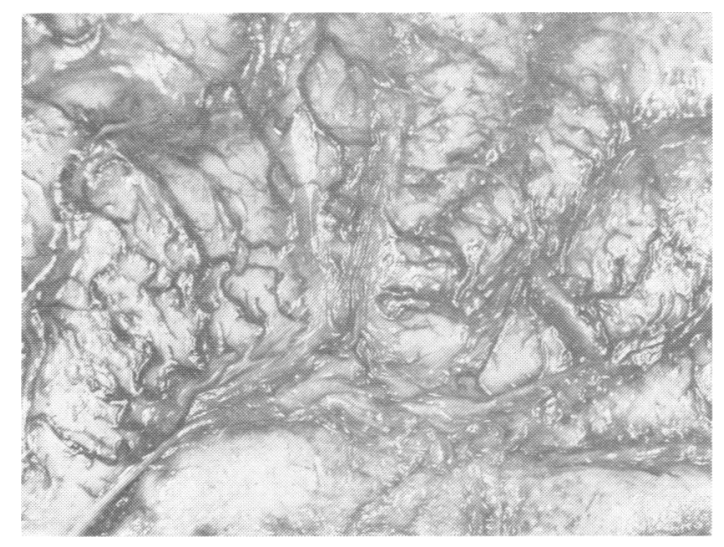

Fig. 4 Surface of the right parieto-occipital region of the brain photographed close-up after fixation. There are very numerous tortuous and engorged vessels in the leptomeninges. 


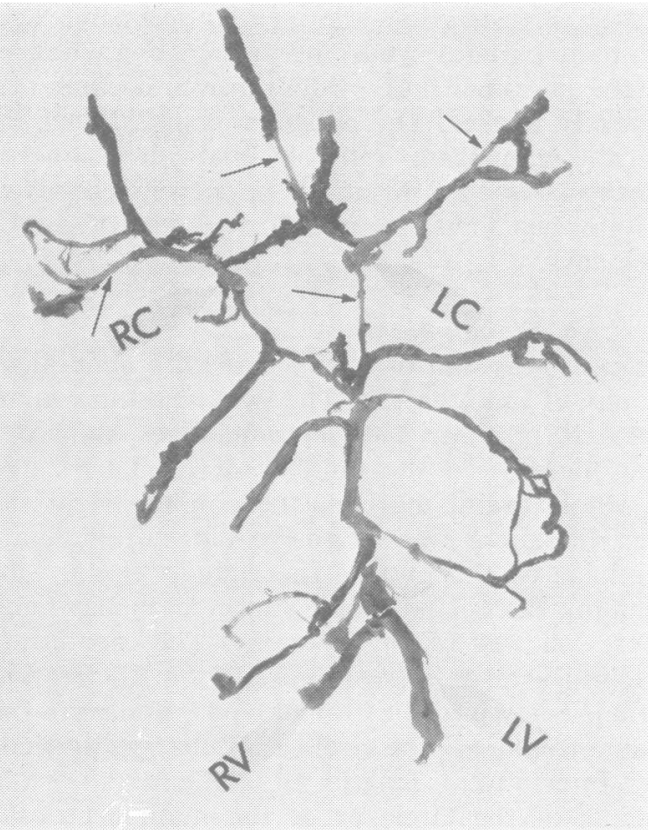

Fig. 5 Circle of Willis dissected from the base of the brain. There are segments (black arrows) of the right anterior, both middle cerebral, and left posterior communicating arteries which are narrowed and whiter than normal. The left vertebral artery is occluded by thrombus ( $R C$ and $L C=$ right and left internal carotid; $R V$ and $L V=$ right and left vertebral arteries).

of engorged vessels throughout the brain in both grey and white matter (Fig. 6). The vessels in the cortex were of small calibre and appeared as pinpoints or hair lines of blood. In the right putamen and globus pallidus and in the white matter of the posterior half of the brain, the engorged vessels were of larger calibre, many of them being about one millimetre in diameter. The sulci contained thick tangles of engorged meningeal vessels (see Fig. 7). Many regions of the brain showed infarction, varying in age from recent to at least several months. Recent infarcts were present in the deep frontal white matter on the right, fornices, white matter of the right posterior temporal lobe, splenium of the corpus callosum, right striate cortex, right caudate nucleus, centromedian region of the thalamus, and basis pontis. There were areas of old infarction in the left middle and inferior frontal gyri and in the right occipital cortex. Extensive recent softening affected the cerebellum, particularly the dorsal aspects of both hemispheres, but spared the deep nuclei. The spinal cord was normal in appearance.

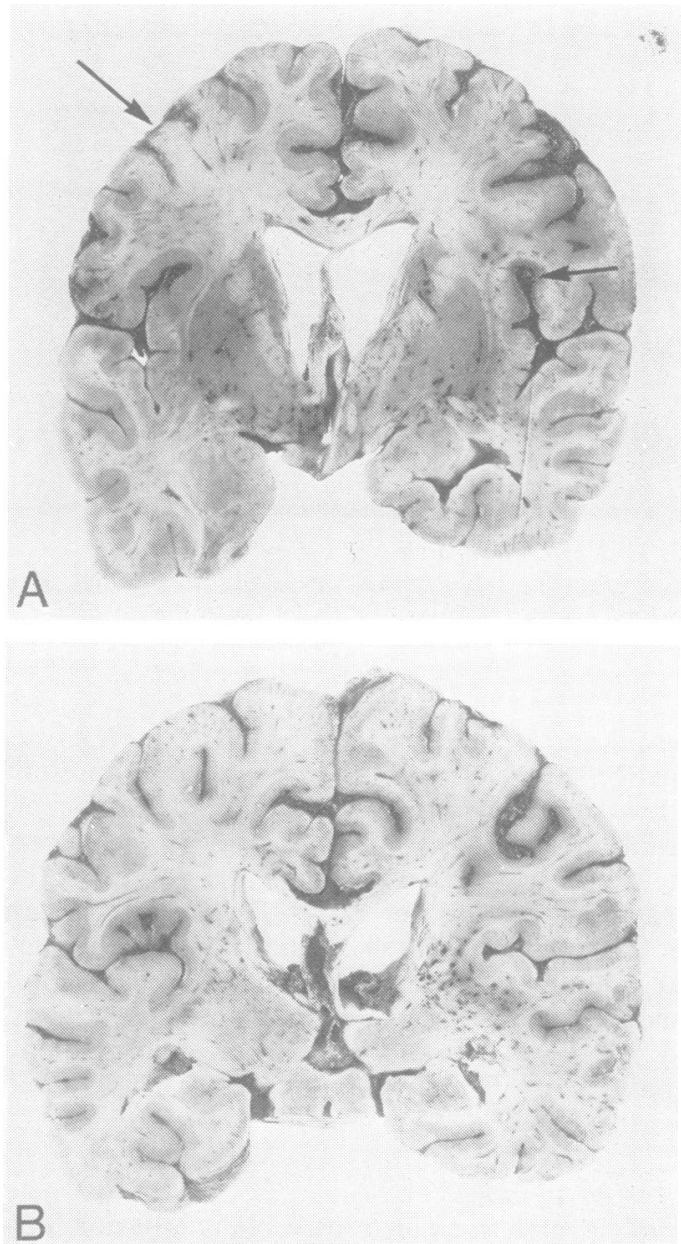

Fig. 6 Coronal slices of the brain at the level of the anterior hypothalamus $(A)$ and of the pulvinar $(B)$. There are many engorged vessels in the deep grey and white matter, more prominently on the right. Note also the areas of old cortical infarction of the left posterior frontal and right insular cortex (arrows).

Histological examination of the brain confirmed the presence of many foci of old and recent ischaemic necrosis. These involved both grey and white matter or only some layers of the cortex. The areas affected are listed above under the macroscopic description of the brain. The basal ganglia and the thalamus, on both sides, contained an increased number of distended small vessels which histologically had the characteristics of capillaries or small veins.

All the individual components of the circle of Willis were examined in longitudinal or in serial transverse sections. The posterior cerebral and 


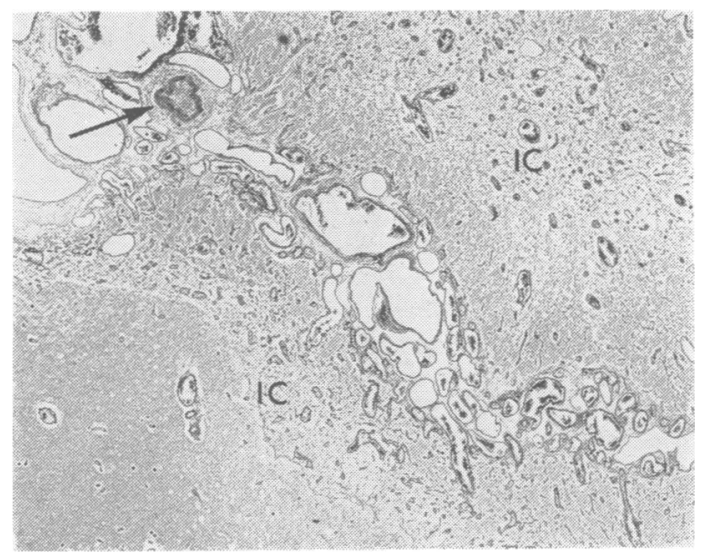

Fig. 7 Section of occipital lobe stained to show reticulin. Areas of cortex showing old infarction are seen (IC) and in the sulcus between them there are many thin-walled leptomeningeal vessels. One occluded artery is sectioned transversely (arrow). $\times 14$.

basilar arteries were relatively normal while anterior cerebral, middle cerebral, and posterior communicating arteries were all histologically abnormal. Accompanying the main arteries, there were "leashes" of numerous thin-walled, tortuous vessels. Communications between the main arteries and these vessels could be seen. In the narrow whitish segments of the cerebral arteries the lumen was completely occluded in many places by a delicate sparsely cellular connective tissue web without evidence of old thrombosis or recanalisation and with very little collagen (Fig. 8). Staining for reticulin fibrils was negative. Cortical arterial branches in several areas of the convexities of frontal, parietal, and occipital lobes showed a similar occlusion of the lumen. Wherever there was occlusion of the arterial lumen, the vessel was contracted and the elastic lamina very markedly thickened and infolded. The elastic lamina of the cerebral arteries generally was markedly abnormal, being patchily thickened (up to $50 \mu \mathrm{m}$ in thickness). The left vertebral artery was occluded by a recent thrombus and showed only a little elastic thickening and intimal changes of atherosclerosis.

The numerous accompanying vessels had thin walls composed of an endothelial lining and a little underlying collagen (Fig. 9). These vessels were not normally formed arteries or veins but, in spite of their thin walls, an elastic lamina was present in some of them. The elastic lamina tended to be straight and was usually not continuous throughout the whole circumference of the vessel. In many of these very thin-walled vessels the elastica was delicate and intermittent as if it were in the early stages of formation.

Both the intracranial and extracranial portions of the internal carotid arteries were examined. They showed thickening and fibrosis of the intima, either diffuse or patchy, and splitting and thickening of the internal elastic lamina, changes compatible with atherosclerosis.

Outside the central nervous system there was some atherosclerosis of the abdominal aorta and of the coronary vessels, and histologically there was patchy myocardial fibrosis. In no other organs were vascular changes seen comparable with those changes described in the cerebral vessels.

Histological examination showed widespread myopathic changes in muscles, more marked in some. They included the presence of fibres of markedly varying diameter, central nuclei, vacuolation, fibre necrosis, and invasion by myophages and splitting of muscle fibres. Muscle fibres with large nuclei and basophilic cytoplasm suggested regenerative activity, and there was an increase of perimysial and, in places, endomysial connective tissue. Blood vessels, intramuscular nerve fibres and muscle spindles appeared normal.

\section{Discussion}

The radiological moya-moya appearance is generally regarded as a sequal to occlusion of the terminal internal carotid and its branches rather than as the primary lesion (Suzuki and Takaku, 1969). The specific features of the obliterative process which stimulates this collateral response remain obscure. However, the relatively large number of case reports of children (see Taveras, 1969) suggests that the potential for such collateral channels may be related to age, and it could be argued that in many of the adults who present the features of moya-moya disease, the disorder of the circulation is in fact longstanding. The time course of obliteration also seems important. Serial angiographic evidence has shown the insidious progression of the process over a number of months in children, while in adults the vessel configuration was unchanged on follow-up angiograms, suggesting that the pathological process had stabilised in adulthood (Suzuki and Takaku, 1969). However, progression of the disease with development of typical moya-moya vessels has been shown angiographically in a 52 year old German woman (Zülch et al., 1974).

The aetiology of moya-moya disease remains obscure. There is little doubt that the fine-vessel collateral response can be stimulated by a number 


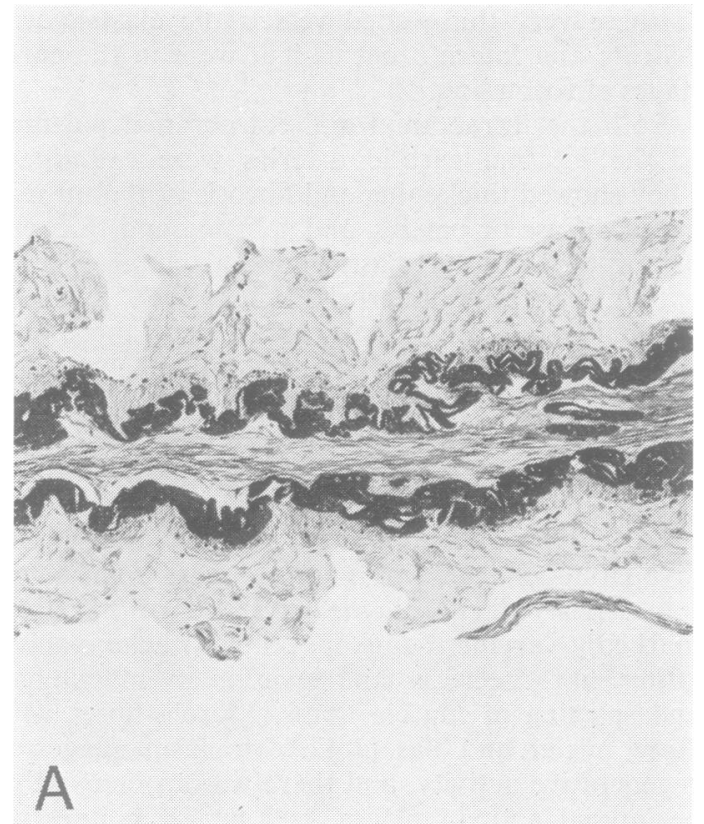

Fig. 8 Longitudinal $(A)$ and transverse $(B)$ sections of the narrow whitish regions of the cerebral arteries shown in Fig. 5. The lumen is occluded by a delicate web of connective tissue. The elastic lamina is irregularly thickened and thrown into elaborate folds. There is no evidence of atheroma or of organisation of old thrombus. Elastic and Van Gieson, $\times 90$.

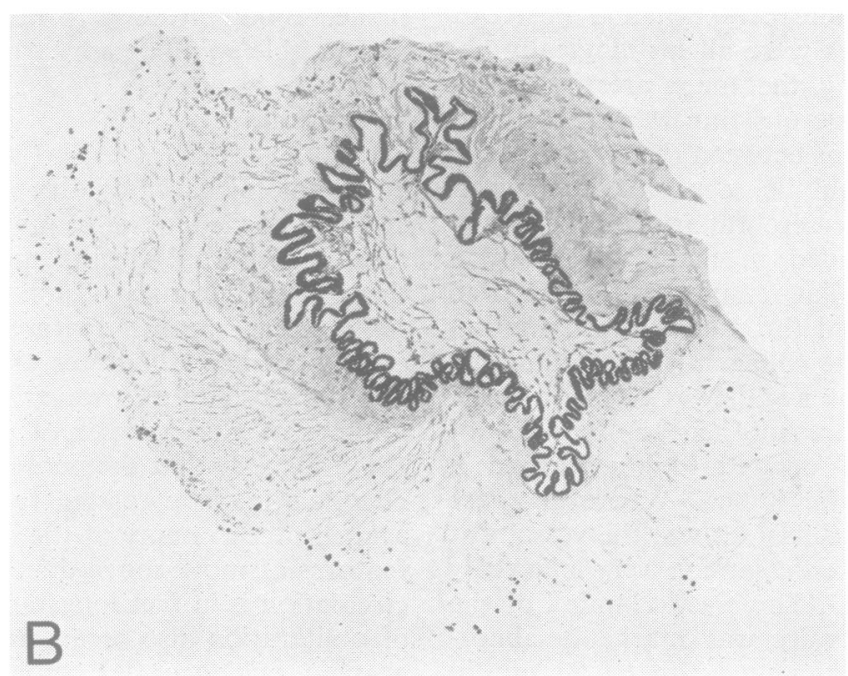

of pathological processes which cause vascular occlusion at the base of the brain. For example, the collateral network has been described in cases of neurofibromatosis with optic nerve glioma (Hilal et al., 1971) stenotic tumours (Rosengren, 1974), basal meningitides-either tuberculous or pyogenic-(Mathew et al., 1970), and associated with Marfan's syndrome and atherosclerosis (Taveras, 1969). Such reports of specific aetiological factors represent a small fraction of the literature and the majority of cases remain "idiopathic."

The cases reported by Poór and Gács (1974) showed atherosclerosis of the carotid arteries but it seems that histological examination of the vessels at the base of the brain was not complete. Although it may be possible that in occasional cases with atherosclerotic disease a collateral system of vessels develops, we do not hold the view that the collateral network appearances is simply a nonspecific radiological syndrome nor that most cases can be explained in terms of known pathological processes. Indeed the necropsy 


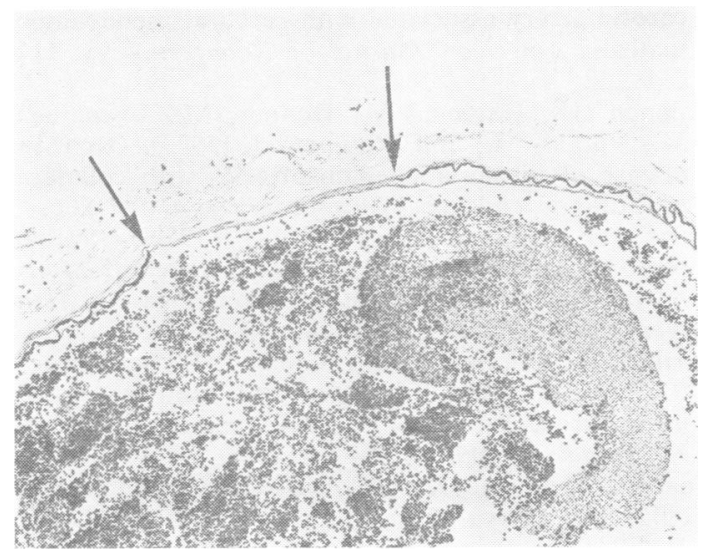

Fig. 9 Transverse section of one of the numerous abnormal leptomeningeal vessels. The vessel wall is very thin in relation to the diameter of the lumen (which contains red blood cells). An elastic lamina is present beneath the endothelium but is absent in the region indicated by the arrows. External to the elastic lamina there is little or no muscle coat. Elastic and Van Gieson, $\times 90$.

studies indicate that this is a cerebrovascular disease of an unfamiliar nature. The relatively high incidence in Japan is inescapable, and unlikely to be the result of differences in the intensity of investigation compared with, for example, the USA. This has led Suzuki and Takaku (1969) to draw attention to the obvious parallel with the other predominantly Japanese vascular disease, Takayasu's arteritis. However, the histological changes in the present case and the other necropsies are quite unlike the inflammatory appearances seen in Takayasu's disease. Neither is there histological evidence for an autoimmune process such as rheumatoid vasculitis or polyarteritis nodosa which are known to involve the cerebral vessels occasionally (Ford and Siekert, 1965). Systemic lupus erythematosus has been reported to cause tapering occlusion of the supraclinoid internal carotid arteries (Trevor et al., 1972) and, histologically, segmental proliferation of the intima has been described (De Morsier and Gaillard, 1959). However, systemic lupus erythematosus has yet to be reported as a cause of moya-moya disease.

Whatever the aetiology, genetic factors seem to play some part since in addition to the racial predisposition there is evidence for familial occurrence. Nishimoto and Takeuchi (1972) described four pairs of affected siblings, and from Europe there are two reports of familial involvement (Levesque et al., 1973; Sogaard and Jorgensen,
1975). Sogaard and Jorgensen (1975) describe a remarkable family of 14 siblings in which three had angiographically proven moya-moya disease, a further eight had severe headaches but were uninvestigated, and the child of one had tuberous sclerosis with episodes of hemiparesis.

The association of moya-moya disease with a type of muscular dystrophy of late onset as in the present case, has, to our knowledge, not been recorded previously. The muscle pathology was characteristically that of a primary muscle disease, no vascular changes comparable with those seen intracranially being present. The association of the two diseases should best be regarded as fortuitous.

Although many cases studied radiologically have been reported as showing the moya-moya characteristics, few of these cases have been examined postmortem, and very few histological descriptions have been found in the literature. The changes which have been reported include the marked prominence of surface vessels (Maki and Nakata, 1965; Halonen et al., 1973) and of deep vessels throughout the brain (Vuia et al., 1970), or occlusion of one or more of the components of the circle of Willis (Kawakita et al., 1965). Some authors have described vessels as hypoplastic generally (Vuia et al., 1970) while others described hypoplasia of one or other constituent of the vessel wall. Nearly all reports include descriptions of an abnormal elastic lamina which was either excessively infolded and thickened (Kawakita et al., 1965; Maki and Nakata, 1965; Vuia et al., 1970), or was intermittent or absent (Harvey and Alvord, 1972; Carlson et al., 1973; Halonen et al., 1973).

In the present case there were numerous discrete regions of the cerebral arteries which were occluded. These discrete arterial occlusions varied in length but were quite separate from each other. The occluded segments of the vessels were narrow and white, and on histological examination the lumen was filled by a delicate connective tissue web, while the elastic lamina was thrown into complex, elaborate, and deep folds, and was patchily thickened. This pathological appearance is quite unlike that caused by atheroma, old thrombosis, inflammatory arteritis, or any other aetiology known to us. The appearances seem to be quite unusual, and the underlying pathogenesis is not known.

Many of the thin-walled vessels forming the leashes accompanying the main arterial branches in the leptomeninges had an unusual appearance in that they possessed an incomplete elastic lamina in spite of a wide lumen relative to the thickness of the wall and the almost complete absence of 
a muscle coat. These abnormal thin-walled vessels could have originated as newly formed vessels growing as a consequence of arterial occlusion. This may be the case particularly in acquired moya-moya secondary to arteritis in chronic meningitis occurring at an early age (Mathew et al., 1970) but is almost unknown in other forms of cerebral arterial occlusion. No evidence of capillary budding was seen in the leptomeningeal vessels in the present case, although the expected glial-mesodermal reaction was observed around infarcted areas of the brain. It seems more likely that the source of the collateral vessels was from smaller pre-existing capillaries or venules, or was part of a vascular overgrowth occurring as a developmental phenomenon. The irregular distribution of elastic and muscle layer is in keeping with either an abnormality of development or arterialisation of small veins or capillaries.

It is evident that a fine vessel collateral response can be stimulated by basal arterial occlusion from known pathological processes. Such rare cases are clearly secondary but the majority of cases of moya-moya disease could be designated as primary. Whatever the pathogenesis, this case seems to indicate that primary "moya-moya disease" should be regarded as a distinct entity from the pathological as well as radiological points of view.

We should like to thank Dr E. A. Kaufmann and Mr C. E. Polkey for permission to publish this case report, Dr J. Dawson for the radiological investigation, and Dr Ruth Harris for the electromyography report. We should also like to thank Miss P. Carter and Mr P. E. Green for the microscopic preparations, and $\mathrm{Mr}$ J. A. Mills for help with the photography.

\section{References}

Busch, H. F. U. (1969). Unusual collateral circulation in a child with cerebral arterial occlusion. Psychiatria, Neurologia, Neurochirurgia, 72, 23-28.

Carlson, C. B., Harvey, F. H., and Loop, J. (1973). Progressive alternating hemiplegia in early childhood with basal arterial stenosis and telangiectasia (moya-moya syndrome). Neurology (Minneapolis), 23, 734-744.

De Morsier, G., and Gaillard, L. A. (1959). Lupus erythémateux disséminé avec artérite généralisée: atrophie granulaire et lésions cérébrales multiples. Schweizer Archiv für Neurologie und Psychiatrie, 84, 258-260.

Ford, R. G., and Siekert, R. G. (1965). Central nervous system manifestations of periarteritis nodosa. Neurology (Minneapolis), 15, 114-120.

Galligioni, F., Andrioli, G. C., Marin, G., Briani, S., and Iraci, G. (1971). Hypoplasia of the internal carotid artery associated with cerebral pseudoangiomatosis. American Journal of Roentgenology, 112, 251-262.

Halonen, H., Halonen, V., Donner, M., Iivanainen, M., Vuolio, M., and Makinen, J. (1973). Occlusive disease of intracranial main arteries with collateral networks in children. Neuropediatrie, 4, 187-206.

Harvey, F. H., and Alvord, E. C. (1972). Juvenile cerebral arteriosclerosis and other cerebral arteriopathies of childhood. Six autopied cases. ActaNeurologica Scandinavica, 48, 479-509.

Hilal, S. K., Solomon, G. E., Gold, A. P., and Carter, S. (1971). Primary cerebral artery occlusive disease in children. Part II. Neurocutaneous syndromes. Neuroradiology, 99, 87-94.

Hoare, A. M., and Keogh, A. J. (1974). Cerebrovascular moyamoya disease. British Medical Journal, 1, 430-432.

Kawakita, Y., Abe, K., Miyata, Y., and Horikoshi, S. (1965). Spontaneous thrombosis of the internal carotid artery in children. Folia Psychiatrica et Neurologica Japonica, 19, 245-255.

Kudo, T. (1968). Spontaneous occlusion of circle of Willis; disease apparently confined to Japanese. Neurology (Minneapolis), 18, 485-496.

Kudo, T. (1971). A report presented at a special symposium in Tokyo. Naika, 28, 465-469. (In Japanese).

Lepoire, J., Tridon, P., Montant, J., Hepner, H. Renard, M., and Picard, L. (1969). Malformations angiomateuses artério-artérielles du système caroti dien. Neurochirurgie, 15, 5-18.

Levesque, M., Bories, J., and Lefebvre, J. (1973) Moya Moya. A propos de six observations. Annal. de Radiologie, 16, 27-40.

Maki, Y., and Nakata, Y. (1965). An autopsy case of hemangiomatous malformation of bilateral internal carotid artery at the base of the brain. Brain Nerve, Tokyo, 17, 764-766. (In Japanese).

Mathew, N. T., Abraham, J., and Chandy, J. (1970). Cerebral angiographic features in tuberculous meningitis. Neurology (Minneapolis), 20, 1015-1023.

Meriwether, R. P., Barnett, H. G., and Echols, D. H. (1976). Moya moya disease as a cause of subarachnoid hemorrhage in a negro patient. Journal of Neurosurgery, 44, 620-622.

Nishimoto, A., and Takeuchi, S. (1972). Moyamoya disease. Abnormal cerebrovascular network in the cerebral basal region. In Handbook of Clinical Neurology, vol. 12, pp. 351-383. Edited by P. J. Vinken and G. W. Bruyn. North-Holland: Amsterdam.

O’Sullivan, D. J. (1973). Cerebrovascular moya moya disease. Proceedings of the Australian Neurological Association, 9, 73-79.

Poór, G., and Gács, G. (1974). The so-called "moyamoya disease". Journal of Neurology, Neurosurgery, and Psychiatry, 37, 370-377.

Rosengren, K. (1974). Moyamoya vessels. Collateral arteries of the basal ganglia. Malignant occlusion of the anterior cerebral arteries. Acta Radiologica, 15, 145-151. 
Simon, J., Sabouraud, O., Guy, G., and Turpin, J. (1968). Un cas de maladie de Nishimoto. A propos d'une maladie rare et bilatérale de la carotide interne. Revue Neurologique, 119, 376-383.

Sogaard, I., and Jorgensen, J. (1975). Familial occurrence of bilateral intracranial occlusion of the internal carotid arteries (moya moya). Acta Neurochirurgica, 31, 245-252.

Suzuki, J., and Takaku, A. (1969). Cerebrovascular "moyamoya" disease. Disease showing abnormal net-like vessels in base of brain. Archives of Neurology (Chicago), 20, 288-299.

Takeuchi, K. (1961). Occlusive disease of the carotid artery. Recent Advances in Research of the Nervous System (Tokyo), 5, 511-543. (In Japanese).

Taveras, J. M. (1969). Multiple progressive intracranial arterial occlusions: a syndrome of children and young adults. American Journal of Roentgenology, 106, 235-268.

Trevor, R. P., Sonderheimer, F. K., Fessel, W. J., and Wolpert, S. U. (1972). Angiographic demonstration of major cerebral vessel occlusion in systemic lupus erythematosus. Neuroradiology, 4, 202-207.

Vuia, O., Alexianu, M., and Gabor, S. (1970). Hypoplasia and destruction of the circle of Willis in a case of atypical cerebral haemorrhage and its relationship to Nashimoto's disease. Neurology (Minneapolis), 20, 361-367.

Zülch, K. J., Dreesbach, H. A., and Eschbach, O. (1974). Occlusion of the middle cerebral artery with the formation of an abnormal arterial collateral system-moyamoya type-23 months later. Neuro radiology, $7,19-24$. 